\title{
A study of field emission from glass spheres, coated with carbon nanotubes
}

\author{
Stephen M. Lyth* and S. R. P. Silva \\ Advanced Technology Institute \\ University of Surrey \\ Guildford, Surrey, UK \\ *s.lyth@surrey.ac.uk \\ Telephone: +44 1481 686083, Fax: +44 148368944
}

\section{Introduction}

Carbon nanotubes have excellent field emission (FE) properties. ${ }^{1-2}$ The geometry and morphology of a surface are crucial aspects of this, and a greater understanding of these properties could lead to the design of more efficient FE devices. We compare the FE properties of a flat, multiwall carbon nanotube (MWNT) layer with those of micron-scale glass spheres, coated with MWNT. We compare this experimental data with simulations and present this as experimental verification of the giant multistage geometric enhancement effect. $^{3}$

\section{Experimental}

A MWNT ink was formed by refluxing MWNT in a concentrated nitric and sulphuric acid mixture, the details of which can be found elsewhere. ${ }^{4}$ This ink was spin-coated onto microwave oxygen plasma-treated indium tin oxide (ITO)-coated glass slides, forming a flat MWNT layer. Then the MWNT ink was mixed with microspheres, and deposited onto an ITO-coated glass slide by pipette. The resulting substrates were baked to remove residual water.

A field simulation of a solitary conducting sphere and of an array of touching spheres on a plane was carried out using Simion $3 \mathrm{D}^{\mathrm{TM}}$ software. The enhancement factors $(\beta)$ were calculated by dividing the electric field (E) at the apex of each sphere by $E$ at the anode. This yielded simulated $\beta$ values of $\beta_{\text {simA }}=3.7$ and $\beta_{\text {simB }}=2.9$ for a single sphere and the array of spheres, respectively.

\section{Results}

Figures 1(a)-1(c) show SEM images of the MWNT coated spheres. It is evident that MWNT adhere well and uniformly to the spheres. Figure 1(b) shows MWNT spanning the gap between spheres. This connects the spheres both electrically and physically. Figure 1(c) shows the nanoscale morphology of the MWNT layer on the glass spheres. This can be compared with the flat MWNT layer in (Fig. 1(d)). It is evident that MWNT on the spherical substrates have a slightly more complex surface morphology than those on flat substrates.
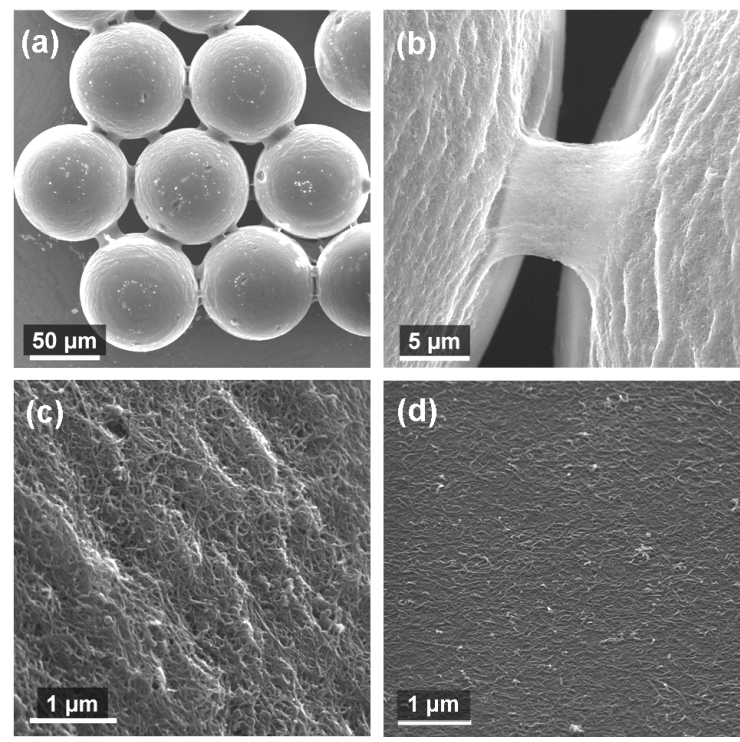

Figure 1: SEM images of the MWNT-coated substrates.

The FE properties were investigated in a diode configuration with a spherical stainless-steel anode. The threshold field $\left(\mathrm{E}_{\mathrm{th}}\right)$ was defined as the macroscopic electric field at which $1 \mathrm{nA}$ was detected. Figure 2(a) shows the FE current versus macroscopic electric field for the MWNT-coated glass spheres and for a flat MWNT film. $\mathrm{E}_{\text {th }}$ was measured as $17.8 \mathrm{~V} / \mu \mathrm{m}$ for the flat film and 3.22 $\mathrm{V} / \mu \mathrm{m}$ for the MWNT-coated spheres. The difference is attributed to the spherical curvature of the emission surface due to the geometry of the glass spheres. Figure 2(b) shows the FE data plotted in Fowler-Nordheim coordinates. Assuming the work function of MWNT to be 5.0 $\mathrm{eV},{ }^{4} \beta$ for the flat MWNT layer $\left(\beta_{\text {flat }}\right)$ was calculated to be 320 . This is higher than expected 
for a totally flat surface $(\beta=1)$, due to the complex geometry of the MWNT layer. $\beta$ for the MWNT-coated spheres $\left(\beta_{\text {total }}\right)$ is 1330 . This is much higher than that of $\beta_{\text {flat, }}$ due to the enhancement caused by the spherical geometry combined with the enhancement of the MWNT layer. These results are summarised in Table 1.
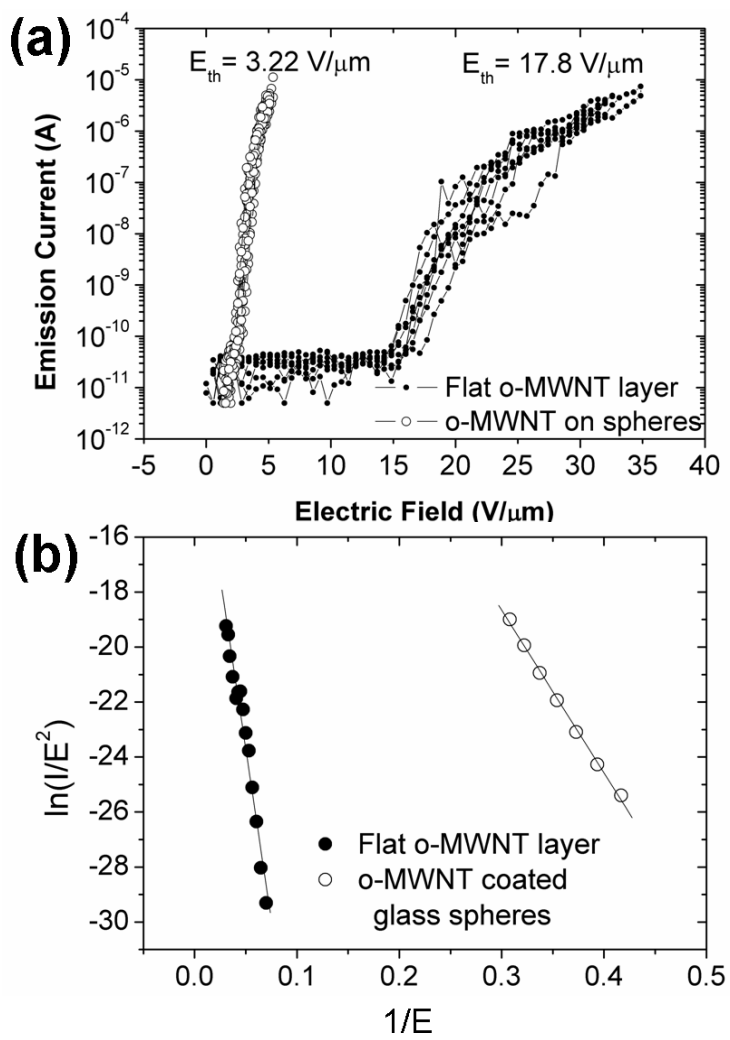

Figure 2(a): Current field data and (b) Fowler Nordheim plots

Assuming a giant multistage enhancement effect, $\beta_{\text {total }}$ should be a product of the $\beta$ values of the spheres $\left(\beta_{\text {sphere }}\right)$ and of the flat MWNT layer, i.e.

$$
\beta_{\text {total }}=\beta_{\text {flat }} \times \beta_{\text {sphere }}
$$

It follows that;

$$
\beta_{\text {sphere }}=\beta_{\text {total }} / \beta_{\text {flat }}=4.2
$$

This value should compare well with the values $\beta_{\mathrm{simA}}=3.7$ and $\beta_{\mathrm{simB}}=2.9$. Although the answer is close, it is an overestimate. If we assume that FE is more likely to come from individual spheres (where screening effects can be ignored), then $\beta_{\text {sphere }}$ would be expected to be closer to $\beta_{\text {simA }}$ than $\beta_{\text {simB }}$. Additionally, the difference in morphology between the flat and curved MWNT layers is not considered. Taking this into account, it would follow that using $\beta_{\text {flat }}$ in Equation [3] would lead to an over estimate of $\beta_{\text {sphere. Therefore, Equation }}$ [3] can be rewritten as;

$$
\beta_{\text {sphere }}<\beta_{\text {total }} / \beta_{\text {flat }}<4.2[4]
$$

Therefore, the experimental results are described closely by the simulated results, providing evidence that multistage field enhancement is a realistic model.

\begin{tabular}{ccc}
\hline & $\mathrm{E}_{\text {th }}(\mathrm{V} / \mu \mathrm{m})$ & $\beta$ \\
\hline Flat MWNT & $\mathbf{1 7 . 8}$ & $\mathbf{3 2 0}$ \\
MWNT spheres & $\mathbf{3 . 2 2}$ & $\mathbf{1 3 3 0}$ \\
Simulated array of spheres & $\mathbf{n} / \mathbf{a}$ & $\mathbf{2 . 9}$ \\
Simulated solitary sphere & $\mathbf{n} / \mathbf{a}$ & $\mathbf{3 . 7}$ \\
Extrapolated value for sphere & $\mathbf{n} / \mathbf{a}$ & $\mathbf{4 . 2}$ \\
\hline Table 1: Summary of numerical data &
\end{tabular}

\section{Conclusion}

In conclusion, MWNT ink was spin-coated onto ITO-coated glass substrates and co-deposited with microspheres onto identical substrates. The FE characteristics of the films were compared and FE from microspheres (compared to flat MWNT films) reduced $\mathrm{E}_{\mathrm{th}}$ from $17.8 \mathrm{~V} / \mu \mathrm{m}$ to $3.22 \mathrm{~V} / \mu \mathrm{m}$. $\beta$ for the flat films and the MWNT-coated spheres was found to be 318 and 1330 , respectively. The $\beta$ factor of an uncoated sphere was extrapolated from these results $\left(\beta_{\text {sphere }}<4.2\right)$, assuming a giant multistage geometric field enhancement effect. This was compared to the simulated $\beta$ of a sphere on a plane (3.7) and found to be in good agreement.

We would like to thank EPSRC (UK) for funding this research via the $\mathrm{CBE}$ and Portfolio Partnership programmes. Thanks are due to Richard Smith for experience with simulation software.

\section{References}

1. A. G. Rinzler, et al. Science 269, 1550 (1995)

2. W. A. Deheer, et al. Science 270, 1179 (1995)

3. J. Y. Huang, et al. APL. 87, 053110 (2005)

4. S. M. Lyth et al. APL 90, 013120 (2007) 